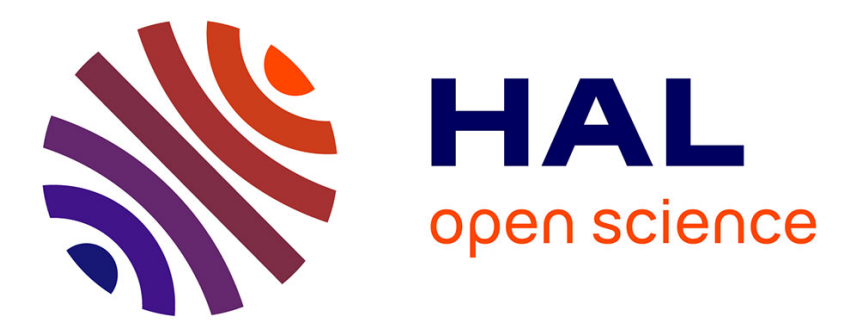

\title{
Exploiting Separators for Guiding VNS
}

\author{
Samir Loudni, Mathieu Fontaine, Patrice Boizumault
}

\section{To cite this version:}

Samir Loudni, Mathieu Fontaine, Patrice Boizumault. Exploiting Separators for Guiding VNS. Electronic Notes in Discrete Mathematics (39), 2012, pp.265-272. hal-01023183

\section{HAL Id: hal-01023183 \\ https://hal.science/hal-01023183}

Submitted on 15 Jul 2014

HAL is a multi-disciplinary open access archive for the deposit and dissemination of scientific research documents, whether they are published or not. The documents may come from teaching and research institutions in France or abroad, or from public or private research centers.
L'archive ouverte pluridisciplinaire HAL, est destinée au dépôt et à la diffusion de documents scientifiques de niveau recherche, publiés ou non, émanant des établissements d'enseignement et de recherche français ou étrangers, des laboratoires publics ou privés. 


\title{
Exploiting Separators for Guiding VNS
}

\author{
Samir Loudni ${ }^{\mathrm{a}}$ Mathieu Fontaine ${ }^{\mathrm{a}}$ Patrice Boizumault ${ }^{\mathrm{a}}$ \\ ${ }^{a}$ Université de Caen Basse-Normandie, UMR 6072 GREYC, F-14032 Caen, \\ France, CNRS, UMR 6072 GREYC, F-14032 Caen, France
}

\begin{abstract}
This paper presents two extensions for DGVNS (Decomposition Guided VNS) method, that exploit both the graph of clusters and separators between these clusters, to efficiently guide the exploration of large neighborhoods in VNS. Experiments performed on challenging instances of the tagSNP selection problem show the appropriateness and the efficiency of our approach.
\end{abstract}

Keywords: VNS Hybrid, Tree decomposition, Separators, Cost Function Network.

\section{Introduction}

Tree decomposition, introduced by Robertson and Seymour [7], aims to decompose a problem into sub-problems (called clusters) constituting an acyclic graph. Each cluster corresponds to a subset of variables that are strongly connected. For large problems that exhibit a highly structured constraints graph, exploiting such structural properties will lead these problems to be solved more efficiently (see $[8,1]$ for complete search methods).

In a recent paper [2] we have introduced DGVNS (Decomposition Guided VNS), a new approach that exploits the graph of clusters obtained from a tree decomposition of the constraints graph, to efficiently guide the exploration of large neighborhoods in VNS. In this paper, we propose two extensions for DGVNS, noted SGVNS (Separator-Guided VNS) and ISGVNS (Intensified SGVNS), 
that exploits both the graph of clusters and the separators between these clusters. Experimental results over challenging instances of the tagSNP selection problem show that (i) our two approaches clearly outperform VNS/LDS+CP [6], and (ii) ISGVNS is very effective compared to DGVNS and SGVNS.

\section{Definitions and Notations}

A Cost Function Network (CFN) is a pair $(X, W)$ where $X=\left\{x_{1}, \ldots, x_{n}\right\}$ is a set of $n$ variables (with a maximum domain size $d$ ) and $W$ is a set of $e$ cost functions (see Fig. 3). Each variable $x_{i} \in X$ has a finite domain $D_{i}$ of values that can be assigned to it. A value $a$ in $D_{i}$ is denoted $\left(x_{i}, a\right)$. For a set of variables $S \subseteq X, D^{S}$ denotes the cartesian product of the domains of the variables in $S$. A complete assignment $t=\left(a_{1}, \ldots, a_{n}\right)$ is an assignment of all variables; on the contrary, it will be called a partial assignment. For a given complete assignment $t, t[S]$ denotes the projection of $t$ over $S$. A cost function $w_{S} \in W$, with scope $S \subseteq X$, is a function $w_{S}: D^{S} \mapsto\left[0, k_{\top}\right]$ where, $k_{\top}$ is a maximum integer cost (finite or not) used to represent forbidden assignments (expressing hard constraints). Costs are combined using the bounded addition defined by $\alpha \oplus \beta=\max \left(k_{\top}, \alpha+\beta\right)$. Solving a CFN consists in finding a complete assignment $t$ minimizing $\oplus_{w_{S} \in W} w_{S}(t[S])$.

Definition 2.1 A tree decomposition of a connected $\mathrm{CFN}$ is a pair $\left(C_{T}, T\right)$ where $T=(I, A)$ is a tree with nodes set $I$ and edges set $A$ and $C_{T}=\left\{C_{i} \mid i \in\right.$ $I\}$ is a family of subsets of $X$ (called clusters [7]) such that: (i) $\cup_{i \in I} C_{i}=X$, (ii) $\forall w_{S} \in W, \exists C_{i} \in C_{T}$ s.t. $S \subseteq C_{i}$, (iii) $\forall i, j, k \in I$, if $j$ is on the path from $i$ to $k$ in $T$, then $C_{i} \cap C_{k} \subseteq C_{j}$.

Definition 2.2 The intersection of two clusters $C_{i}$ and $C_{j}$ is called a separator, and noted $\operatorname{sep}\left(C_{i}, C_{j}\right)$.

Definition 2.3 A graph of clusters for a tree decomposition $\left(C_{T}, T\right)$ is an undirected graph $G=\left(C_{T}, E\right)$ that has a vertex for each cluster $C_{i} \in C_{T}$, and there is an edge $\left(C_{i}, C_{j}\right) \in E$ when $\operatorname{sep}\left(C_{i}, C_{j}\right) \neq \emptyset$.

\section{Decomposition Guided VNS}

DGVNS (Decomposition Guided VNS [2]) extends VNDS [4], by exploiting the graph of clusters in order to build neighborhood structures enabling a better diversification. DGVNS uses neighborhood structures $N_{k, i}$, where $k$ is the neighborhood dimension and $C_{i}$ is the cluster the variables will be selected in. Fig. 1 (left) depicts the pseudo-code of DGVNS. It starts from an initial solution $S$ which is randomly generated (function genInitSol, line 5). A subset of $k$ 
Function DGVNS $\left(X, P, k_{\text {init }}, k_{\max }, \delta_{\max }\right)$;

(1) begin

(2) let $G$ be the constraints graph of $P$;

(3) let $\left(C_{T}, T\right)$ a tree decomposition of $G$;

(4) $\quad$ let $C_{T}=\left\{C_{1}, C_{2}, \ldots, C_{p}\right\}$;

(5) $S \leftarrow$ genInitSol();

(6) $k \leftarrow k_{\text {init }}, i \leftarrow 1$;

(7) while $\left(k<k_{\max }\right) \wedge$ notTimeOut do

(8) $\quad C_{s} \leftarrow$ CompleteCluster $\left(C_{i}\right)$;

(9) $\quad \mathcal{X}_{\text {un }} \leftarrow$ Hneighborhood $\left(C_{s}, N_{k, i}, S\right)$;

(10) $\mathcal{A} \leftarrow S \backslash\left\{\left(x_{i}, a\right) \mid x_{i} \in \mathcal{X}_{u n}\right\}$;

(11) $\quad S^{\prime} \leftarrow \operatorname{Rebuild}\left(\mathcal{A}, \mathcal{X}_{u n}, \delta_{\text {max }}, S\right)$;

(14)

(15) end

ChangeNeighborDGVNS $\left(S, S^{\prime}, k, i\right)$ endwhile

return $S$
Function $\operatorname{ISGVNS}\left(X, P, k_{\text {init }}, k_{\max }, \delta_{\max }\right)$;

(1) begin

(2) let $G$ be the constraints graph of $P$;

(3) let $\left(C_{T}, T\right)$ a tree decomposition of $G$;

(4) $\quad$ let $C_{T}=\left\{C_{1}, C_{2}, \ldots, C_{p}\right\}$;

(5) $S \leftarrow$ genInitSol();

(6) $\quad k \leftarrow k_{\text {init }}, i \leftarrow 1$;

(7) $T_{\text {List }} \leftarrow \emptyset, P_{\text {List }} \leftarrow \emptyset$;

(8) While $\left(k<k_{\max }\right) \wedge$ notTimeOut do

(9) $\quad C_{s} \leftarrow$ CompleteCluster $\left(C_{i}\right)$;

(10) $\mathcal{X}_{\text {un }} \leftarrow$ Hneighborhood $\left(C_{s}, N_{k, i}, S\right)$;

(11) $\mathcal{A} \leftarrow S \backslash\left\{\left(x_{i}, a\right) \mid x_{i} \in \mathcal{X}_{u n}\right\}$;

(12) $\quad S^{\prime} \leftarrow \operatorname{Rebuild}\left(\mathcal{A}, \mathcal{X}_{u n}, \delta_{\text {max }}, S\right)$;

(16) end
ChangeNeighborISGVNS $\left(S, S^{\prime}, k, i, C_{s}\right)$

endwhile

return $S$

Fig. 1. Pseudo-codes of DGVNS (left) and ISGVNS (right).

variables $C_{s}$ is randomly selected among conflicted ones by the neighborhood heuristic Hneighborhood (line 9). A partial assignment $\mathcal{A}$ is generated from the current solution $S$ by unassigning the $k$ selected variables (line 10). Then, unassigned variables are rebuilt (line 11) by a partial tree search LDS [5] combined with Constraint Propagation (see [6] for more details). The search stops when the maximal dimension size $k_{\max }$ is reached or the TimeOut (line 7 ).

DGVNS favors moves on regions that are closely linked. The concept of cluster embodies this criterion, because of its size, and by the strong connection of the variables it contains. The $k$ variables to be unassigned are selected in a same cluster $C_{i}$. If $\left(k>\left|C_{i}\right|\right)$, then the set of potential variables $C_{s}$ is completed by adding the clusters $C_{j}$ adjacent to $C_{i}$ in order to take into account the topology of the graph of clusters (function CompleteCluster (line 8)). So, the neighborhood structure $N_{k, i}$ is constituted by the set of all subsets of $k$ variables among $C_{s}$ (line 9 ).

The aim of diversification is to sample a large number of different regions, in order to explore the whole search space, and to locate the region containing the global optimum. To perform a better diversification, DGVNS considers successively all the $C_{i}$ (see procedure ChangeNeighborDGVNS, Fig. 2). Let $p$ be the total number of clusters in $C_{T}$, succ a successor function ${ }^{1}$, and $N_{k, i}$ the current neighborhood structure: if the rebuild step finds a better solution $S^{\prime}$ in the neighborhood of $S$ (line 2), then $S^{\prime}$ becomes the current solution (line 3), $k$ is reset to $k_{\text {init }}$ (line 4 ), and the next cluster is considered (line $5)$. Otherwise, DGVNS looks for improvements in $N_{(k+1) \text {,succ }(i)}$ (neighborhood structure where $(k+1)$ variables of $C_{s}$ will be unassigned (line 7$)$ ).

$\overline{1_{\operatorname{succ}}(i)}=i+1$ if $i<p$, otherwise $\operatorname{succ}(p)=1$. 
First, diversification performed by moving from cluster $C_{i}$ to cluster $C_{\text {succ(i) }}$ is necessary. Experiments we performed have shown that remaining in the same cluster leads to lower improvements: selecting a new cluster enables to visit new parts of the search space. Second, when a local minimum is got in the current neighborhood, moving from $k$ to $(k+1)$ will also provide some diversification by enlarging the neighborhood size.

\section{Exploiting Separators to Guide VNS}

Variables occurring in a separator $\operatorname{sep}\left(C_{i}, C_{j}\right)$ constitute "key-points" of the problem because their re-assignments (in the rebuild-step) will directly impact the variables of both $C_{i}$ and $C_{j}$. SGVNS (Separator-Guided VNS) exploits variables occurring in separators in order to guide the diversification effort towards clusters that are more likely to lead to larger improvements.

Let $S^{\prime}$ be a new solution better than the current solution $S$ and $C_{i}$ be the current cluster. Let $V_{c}$ be the set of all the variables that have been re-assigned to obtain $S^{\prime}$. Let $C_{w}$ be the set of clusters $C_{j}$ such that $\operatorname{sep}\left(C_{i}, C_{j}\right)$ shares at least one variable with $V_{c}$ (except those of $C_{s}{ }^{2}$ ). DGVNS performs diversification by considering $C_{\text {succ (i) }}$ as the next cluster (see Section 3). SGVNS performs diversification by considering successively the clusters $C_{j} \in C_{w}$. These clusters are more appropriate because they contain at least one re-assigned variable in their separators and therefore are more likely to lead to larger improvements.

ISGVNS (Intensified SGVNS) aims to intensify the exploration around the re-assigned variables. To achieve this goal, a propagation list endowed with a dynamic tabu list are used. The first list manages the set of candidate clusters to be examined after each improvement, while the second list ensures that variables involved in the selection of these candidate clusters (i.e. variables of $V_{c}$ ) will not be considered in $N_{k, i}$ by the function Hneighborhood. Fig. 2 (right) depicts the pseudo-code of procedure ChangeNeighborISGVNS. It uses a propagation list $P_{\text {List }}$ and a dynamic tabu list $T_{\text {List }}$ of size $L$.

The intensification effort of ISGVNS is performed using a propagation list. As for SGVNS, $V_{c}$ is the set of all variables that have been re-assigned (line 4 ), and $C_{w}$ is the set of clusters $C_{j}$ having at least one re-assigned variable in a separator (line 5). Contrary to SGVNS, each cluster $C_{j} \in C_{w}$ is added to $P_{\text {List }}$ (line 7 ), and each variable $x \in V_{c}$ is made tabu for the next $L$ iterations (line 9). The value of $L$ is set to the size of $P_{\text {List }}$. This prevents re-assigning $x$ until all the clusters $C_{j} \in C_{w}$ of $P_{\text {List }}$ have been considered. Finally, the next cluster to be considered is selected from $P_{\text {List }}$, if it is not empty (lines 13 and 14). Otherwise, the successor of $C_{i}$ in $C_{T}$ is considered (line 16).

2 This prevents selecting clusters already considered in the current neighborhood $N_{k, i}$. 
Procedure ChangeNeighborDGVNS $\left(S, S^{\prime}, k, i\right)$;

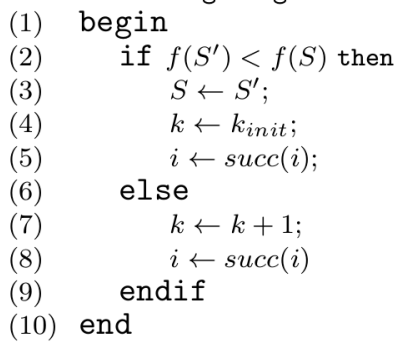

Procedure ChangeNeighborIsgvns $\left(S, S^{\prime}, k, i, C_{s}\right)$;

(1) begin

(2) if $f\left(S^{\prime}\right)<f(S)$ then

(3) $\quad S \leftarrow S^{\prime} ; k \leftarrow k_{\text {init }}$;

(4) $\quad V_{c} \leftarrow\left\{x \mid S^{\prime}[x] \neq S[x]\right\}$;

(5) $\quad C_{w} \leftarrow\left\{C_{j} \mid C_{j} \notin C_{s} \wedge C_{j} \cap V_{c} \neq \emptyset\right\}$;

(6) for each $C_{i} \in C_{w}$ do

(7) insert $i$ in $P_{\text {List }}$

(8) endfor

(9) make tabu each $x \in V_{c}$;

(10) else

(11)

(12)

(13)

(14)

(15)

(16)

(17)

(18) end else

$k \leftarrow k+1$

endif

if $P_{\text {List }}$ is not empty then

else

$i \leftarrow P_{\text {List. }}$.next

$i \leftarrow \operatorname{succ}(i)$

endif

Fig. 2. Steps of neighborhood change for DGVNS (left) and ISGVNS (right).

ISGVNS enforces a balance between intensification and diversification. As long as no improvement is made, ISGVNS behaves as DGVNS by considering successively all the $C_{i}$. But, when a solution is improved, ISGVNS switches to an intensification scheme until all clusters of $P_{\text {List }}$ have been examined.

\section{The tagSNP Selection Problem}

The selection of tagSNPs has became a very active area of research in genotyping [3]. A Single Nucleotide Polymorphism (SNP) is a DNA sequence variation occurring when a single nucleotide - A, T, C or G - in the genome differs between members of a biological species or paired chromosomes in an individual. SNPs act as biological markers that may help predict risk of developing particular diseases. The tagSNP problem consists in selecting a small subset of SNPs, called tagSNPs, that captures most of the genetic information.

A correlation measure $r^{2}$ between any pair of SNPs has been introduced in [3]. A tagSNP $p_{i}$ is said to be representative of another SNP $p_{j}$ if $p_{i}$ and $p_{j}$ are considered as enough correlated (i.e., $r^{2}\left(p_{i}, p_{j}\right) \geq r_{0}$, where $r_{0}$ is a minimum threshold). The tagSNP problem consists in selecting a minimum number of SNPs such that all SNPs are covered. Other criteria [8] can also be considered: (i) maximizing the weighted coverage sum of unselected SNPs and (ii) maximizing the dispersion between selected SNPs (i.e., tagSNPs).

This problem is modeled as a binary CFN (see Section 2). Two variables $i_{s}$ and $i_{r}$ are associated to each SNP $p_{i}: i_{s}$ is a boolean variable that indicates if $p_{i}$ is a tagSNP; $i_{r}$ is a variable representing the tagSNP covering $p_{i}$ (its 
domain is the set of neighbors of $p_{i}$ together with $p_{i}$ itself). For each pair of SNPs $\left(p_{i}, p_{j}\right)$ s.t. $r^{2}\left(p_{i}, p_{j}\right) \geq r_{0}$, the following (hard) constraints are enforced: $i_{s} \Rightarrow\left(i_{r}=p_{i}\right)$ and $\left(i_{r}=p_{j}\right) \Rightarrow j_{s}$. Such constraints are encoded as binary cost functions (with 0 or $k_{\top}$ costs). Preferences (i) and (ii) are respectively captured by unary and binary cost functions (see [8] for more details).

\section{$6 \quad$ Experiments}

Experimental protocol. We have selected ten challenging instances derived from human chromosome-1-data ${ }^{3}$ with $r_{0}=0.5$. Seven instances are of medium size, while the three other ones are large ones. Fig. 3 (leftmost column) gives the characteristics of each instance: its name, its number of variables $(n)$, its number of cost functions $(e)$ and its maximum domain size $(d)$. We used the following parameter settings, which are the best values found (see [6] for more details): $k_{\min }=4, k_{\max }=n$ and $\delta_{\max }=3$. TimeOut was set to 2 hours (resp. 4 hours) for medium-size (resp. large-size) instances. A set of 50 runs per instance has been performed using an AMD-Opteron with $2.1 \mathrm{GHz} \mathrm{CPU}$ and $256 \mathrm{~GB}$ of RAM. All search strategies have been implemented in $\mathrm{C}++$. For each instance and each method, Fig. 3 reports the number of successful runs to reach the optimum, the average number of performed iterations, the average CPU time (in seconds) for the successful runs, the average cost and the best cost (between parentheses) for unsuccessful runs. Four methods are compared: DGVNS, SGVNS, ISGVNS and VNS/LDS+CP [6] (an instance of VNDS [4] which uses neighborhood structures $N_{k}$ of dimension $k$ ). Tree decompositions are built using the Maximum Cardinality Search (MCS) heuristic [9].

Impact of the tree decomposition. For medium-size instances, DGVNS, SGVNS and ISGVNS clearly outperform VNS/LDS+CP (see Fig. 3). The three methods reach the optimum on each run. VNS/LDS+CP gets the same success rates on two instances, but is on average 3 times slower. On instances \#3792 and \#8956, VNS/LDS+CP gets successful runs only very few times. It remains less competitive both in terms of successful runs and CPU times on the other instances. On large-size instances, VNS/LDS+CP never finds the optimum.

Impact of the separators. Fig. 3 also compares SGVNS with DGVNS. For medium-size instances, SGVNS and DGVNS reach the optimum on each run. However, SGVNS is faster on three instances, and slower on four instances. For large-size instances, DGVNS clearly outperforms SGVNS. For instance \#17034, the success rate is improved about $16 \%$ (from $66 \%$ to $82 \%$ ), the mean deviation from the optimum decreases from $1.43 \%$ to $0.63 \%$, and DGVNS is 4 times faster than SGVNS. These results show that SGVNS is competitive for medium-size

3 http://www. costfunction. org/benchmark 


\begin{tabular}{|c|c|c|c|c|c|}
\hline Instance & Method & Succ. & Iter & Time & Avg \\
\hline \multirow{4}{*}{$\begin{array}{l}\# 3792 \\
n=528, d=59 \\
e=12,084 \\
S^{*}=6,359,805\end{array}$} & DGVNS & $50 / 50$ & 1,248 & 954 & $6,359,805$ \\
\hline & SGVNS & $50 / 50$ & 1,240 & 1,033 & $6,359,805$ \\
\hline & ISGVNS & $50 / 50$ & 1,192 & 853 & $6,359,805$ \\
\hline & VNS/LDS+CP & $15 / 50$ & 5,713 & 2,806 & $6,359,856$ \\
\hline \multirow{4}{*}{$\begin{array}{l}\# 4449 \\
n=464, d=64 \\
e=12,540 \\
S^{*}=5,094,256\end{array}$} & DGVNS & $50 / 50$ & 1,107 & 665 & $5,094,256$ \\
\hline & SGVNS & $50 / 50$ & 1,113 & 661 & $5,094,256$ \\
\hline & ISGVNS & $50 / 50$ & 1,0512 & 675 & $5,094,256$ \\
\hline & VNS/LDS+CP & $48 / 50$ & 5,181 & 2,616 & 5,094256 \\
\hline \multirow{4}{*}{$\begin{array}{l}\# 8956 \\
n=486, d=106 \\
e=20,832 \\
S^{*}=6,660,308\end{array}$} & DGVNS & $50 / 50$ & 1,477 & 4,911 & $6,660,308$ \\
\hline & SGVNS & $50 / 50$ & 1,513 & 5,483 & $6,660,308$ \\
\hline & ISGVNS & $50 / 50$ & 1,457 & 4,118 & $6,660,309$ \\
\hline & VNS/LDS+CP & $12 / 50$ & 4776 & 8,665 & 6,660327 \\
\hline \multirow{4}{*}{$\begin{array}{l}\# 9319 \\
n=562, d=58 \\
e=14,811 \\
S^{*}=6,477,229\end{array}$} & DGVNS & $50 / 50$ & 818.7 & 788 & $6,477,229$ \\
\hline & SGVNS & $50 / 50$ & 797 & 500 & $6,477,229$ \\
\hline & ISGVNS & $50 / 50$ & 923 & 672 & $6,477,229$ \\
\hline & VNS/LDS+CP & $47 / 50$ & 6,171 & 2,434 & $6,477,229$ \\
\hline \multirow{4}{*}{$\begin{array}{l}\# 15757 \\
n=342, d=47 \\
e=5,091 \\
S^{*}=2,278,611\end{array}$} & DGVNS & $50 / 50$ & 511 & 60 & $2,278,611$ \\
\hline & SGVNS & $50 / 50$ & 525 & 104 & $2,278,611$ \\
\hline & ISGVNS & $50 / 50$ & 527 & 80 & $2,278,611$ \\
\hline & VNS/LDS+CP & $50 / 50$ & 2,800 & 229 & $2,278,611$ \\
\hline \multirow{4}{*}{$\begin{array}{l}\# 16421 \\
n=404, d=75 \\
e=12,138 \\
S^{*}=3,436,849\end{array}$} & DGVNS & $50 / 50$ & 1,688 & 2,673 & $3,436,849$ \\
\hline & SGVNS & $50 / 50$ & 1,587 & 2,025 & $3,436,849$ \\
\hline & ISGVNS & $50 / 50$ & 4,240 & 5,863 & $3,436,849$ \\
\hline & VNS/LDS+CP & $37 / 50$ & 5,095 & 3,146 & $3,436,924$ \\
\hline \multirow{4}{*}{$\begin{array}{l}\# 16706 \\
n=438, d=30 \\
e=6,321 \\
S^{*}=2,632,310\end{array}$} & DGVNS & $50 / 50$ & 1,167 & 153 & $2,632,310$ \\
\hline & SGVNS & $50 / 50$ & 888 & 159 & $2,632,310$ \\
\hline & ISGVNS & $50 / 50$ & 872 & 89 & $2,632,310$ \\
\hline & VNS/LDS+CP & $50 / 50$ & 5,494 & 629 & $2,632,310$ \\
\hline \multirow{4}{*}{$\begin{array}{l}\# 10442 \\
n=908, d=76 \\
e=28,554 \\
S^{*}=21,591,913\end{array}$} & DGVNS & $50 / 50$ & 2,264 & 4,552 & $21,591,913$ \\
\hline & SGVNS & $50 / 50$ & 2,496 & 7,153 & $21,591,913$ \\
\hline & ISGVNS & $50 / 50$ & 2,395 & 7,291 & $21,591,913$ \\
\hline & VNS/LDS+CP & $0 / 50$ & 5,887 & - & $22,778,811(22,490,938)$ \\
\hline \multirow{4}{*}{$\begin{array}{l}\# 14226 \\
n=1,058, d=95 \\
e=36,801 \\
S^{*}=25,665,437\end{array}$} & DGVNS & $46 / 50$ & 1,802 & 7,606 & $25,688,751$ \\
\hline & SGVNS & $40 / 50$ & 1,776 & 7,646 & $25,805,242$ \\
\hline & ISGVNS & $50 / 50$ & 1,818 & 9,596 & $25,665,437$ \\
\hline & VNS/LDS+CP & $0 / 50$ & 4,941 & - & $28,299,904(26,830,579)$ \\
\hline \multirow{4}{*}{$\begin{array}{l}\# 17034 \\
n=1142, d=123 \\
e=47,967 \\
S^{*}=38,318,224\end{array}$} & DGVNS & $41 / 50$ & 2,098 & 8,900 & $38,563,232$ \\
\hline & SGVNS & $33 / 50$ & 1,825 & 10,212 & $38,869,514$ \\
\hline & ISGVNS & $36 / 50$ & 1,857 & 10,579 & $38,746,957$ \\
\hline & VNS/LDS+CP & $0 / 50$ & 3,315 & - & $41,352,709(39,850,974)$ \\
\hline
\end{tabular}

Fig. 3. Comparison on medium-size and large-size instances.

instances, and less effective for large-size ones.

For medium-size instances, ISGVNS is faster than DGVNS on four instances, slower on two instances, and similar on instance \#4449. For large-size instances, ISGVNS performs better than DGVNS on instance \#14226, and worse on instance \#17034. Both methods obtain the same success rates on instance \#10442, but DGVNS remains faster. These results show that ISGVNS is more effective than DGVNS, and confirm the importance of exploiting separators.

For medium-size instances, ISGVNS and SGVNS obtain the same success rates. However, ISGVNS is faster than SGVNS on four instances. For large-size 
instances, ISGVNS improves the success rates on two instances. These results highlight the importance of the propagation list to enforce a tradeoff between intensification and diversification.

\section{Conclusions}

We have proposed two extensions of DGVNS that exploit both the graph of clusters and the separators to efficiently guide VNS. Experimental results over challenging instances of the tagSNP selection problem show that SGVNS and ISGVNS clearly outperform VNS/LDS+CP, and ISGVNS is very effective compared to DGVNS and SGVNS. We are currently parallelizing the exploration of clusters.

Acknowledgements. This work is partly supported by the ANR (French Research National Agency) funded project FiCOLOFO ANR-10-BLA-0214.

\section{References}

[1] Dechter, R. and J. Pearl, Tree clustering for constraint networks, Artif. Intell. 38 (1989), pp. 353-366.

[2] Fontaine, M., S. Loudni and P. Boizumault, Guiding VNS with tree decomposition, in: ICTAI (2011), pp. 505-512.

[3] Gopalakrishnan, S. and Z. Qin, TagSNP selection based on pairwise LD criteria and power analysis in association studies, in: Pacific Symposium on Biocomputing (2006), pp. 511-522.

[4] Hansen, P., N. Mladenovic and D. Perez-Brito, Variable neighborhood decomposition search, Journal of Heuristics 7 (2001), pp. 335-350.

[5] Harvey, W. D. and M. L. Ginsberg, Limited discrepancy search, in: IJCAI'95, 1995, pp. 607-615.

[6] Loudni, S. and P. Boizumault, Combining VNS with constraint programming for solving anytime optimization problems, EJOR 191 (2008), pp. 705-735.

[7] Robertson, N. and P. Seymour, Graph minors. ii. algorithmic aspects of treewidth, J. Algorithms 7 (1986), pp. 309-322.

[8] Sánchez, M., D. Allouche, S. de Givry and T. Schiex, Russian doll search with tree decomposition, in: IJCAI, 2009, pp. 603-608.

[9] Tarjan, R. E. and M. Yannakakis, Simple linear-time algorithms to test chordality of graphs, test acyclicity of hypergraphs, and selectively reduce acyclic hypergraphs, SIAM J. Comput. 13 (1984), pp. 566-579. 